\title{
Percutaneous vertebroplasty
}

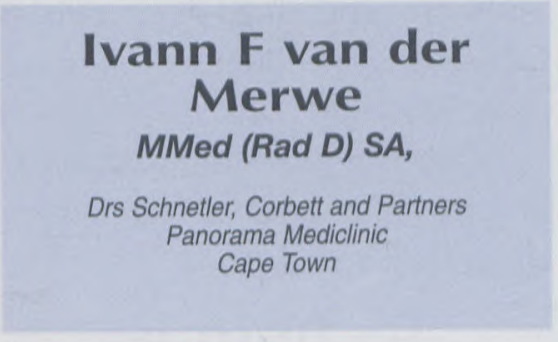

\section{Abstract}

Percutaneous vertebroplasty is a safe and successful method for the treatment of vertebral lesions due to osteoporotic fractures, metastatic lesions or haemangiomas. The procedure involves the percutaneous injection of polymethyl methacrylate (PMMA) into a pathological vertebral body. The technique is described in detail together with potential complications.

Strict adherence to selection criteria, a multidisciplinary approach and the need for excellent imaging equipment are stressed.

\section{Introduction}

Percutaneous vertebroplasty has been performed in France by interventional radiologists since 1984 and involves the percutaneous injection of polymethyl methacrylate (PMMA) into a pathological vertebral body. Currently the same procedure is also being used for the treatment of metastatic lesions in different parts of the skeleton (e.g. the acetabulum), but for the purposes of this article I will confine the discussion to the treatment of vertebral lesions.

The first cases in France were specifically for the treatment of aggressive or symptomatic vertebral haemangiomas, but in Europe the procedure is currently being used mainly for the treatment of primary and secondary malignancies of the vertebrae. In the USA the procedure is more commonly used in the treatment of osteoporotic vertebral fractures than in malignant lesions. This is in part because in the USA it is largely a patient-driven procedure, with the patients having Internet 'savvy' and referring themselves directly. In 1998 there were $10-12$ physicians performing the procedure in the USA; by 2001, 500 interventional radiologists had been trained and were performing percutaneous vertebroplasties.

The procedure is minimally invasive and leads to marked reduction or cure of pain together with the mechanical strengthening of the bone. It is usually done as an outpatient procedure with a success rate of between $80 \%$ and $90 \%{ }^{2}$ The PMMA leads to the stabilisation of the bone fragments. It reaches a temperature of $60-70^{\circ} \mathrm{C}$ on setting, the same temperature reached during the use of radio frequency ablation in the treatment of metastatic deposits. ${ }^{3}$ The result is that this heat generated also leads to lysis of underlying tumour cells.

Vertebroplasty requires a multidisciplinary approach and strict selection criteria are needed to ensure the safety and success of the procedure.

Vertebroplasty must not be mistaken for kyphoplasty where a balloon is first inserted into the collapsed vertebra to try to restore some height before cement is injected.

\section{Indications}

\section{Osteoporotic vertebral fractures}

Sixty per cent of postmenopausal women have osteopenia, of whom $40 \%$ will develop osteoporotic fractures, with between 400000 and 500000 such fractures occurring annually in the USA. Approximately one-third of these patients develop chronic pain of life-altering severity that is unresponsive to conservative management. ${ }^{4,5}$ Vertebral fractures are associated with increased morbidity and mortality and may lead to loss of the patient's independence. They are a leading cause of admissions to nursing homes. Conservative management may be complicated by pneumonia, DVT and pulmonary emboli.

Although pain may last for months a number of patients respond well to conservative management and therefore vertebroplasty should not be attempted before a trial of conservative management has been attempted. The duration obviously ranges with individual patients but a timespan of $2-3$ weeks is currently being used. However, current findings are that patients who have had a fracture previously treated by vertebroplasty, frequently refuse even to attempt conservative therapy when they have another fracture.

\section{Malignancies}

Lymphoma, myeloma and metastatic lesions are the most com- 
monly treated vertebral malignancies. These cause pain by infiltration and due to the collapse of the vertebra.

Although radiation has been used up to now this does not give the same rapid pain relief or stability as one would get with vertebroplasty. It is currently being used in conjunction with vertebroplasty in some centres.

Conservative management of malignant lesions has the same possible complications as can be expected during conservative management of osteoporotic fractures, while surgery has a much higher morbidity, mortality and cost in patients who often have limited life expectancy. By comparison vertebroplasty leads to partial or complete pain reduction in $80-90 \%$ of patients within the first 72 hours. ${ }^{2.6}$ In addition surgery is contraindicated when multiple levels are affected.

The clearest indications for vertebroplasty are in patients complaining of severe, focal and mechanical back pain related to neoplastic vertebral collapse, without epidural involvement.

\section{Haemangiomas}

Painful destructive vertebral haemangiomas (VH) are very rare. Surgical alternatives to vertebroplasty are invasive and require hardware. In these cases vertebroplasty can be considered as an embolisation with bone cement.

Radiological signs of aggressiveness include: (i) progressive involvement of the vertebral body; (ii) extension to the neural arch; (iii) collapse of the vertebral body; and ( $i v)$ increasing soft tissue mass.

\section{Pre-procedural work up}

Vertebroplasty needs a multidisci- plinary approach involving endocrinologists, oncologists, orthopaedic and neurosurgeons. The patients need a thorough neurological evaluation prior to the procedure. It is critical to ensure that the pain is related to the specific vertebral compression and this must be confirmed by clinical evaluation under screening prior to the procedure. Identification of the collapsed vertebra under screening followed by direct pressure should replicate the pain the patient is experiencing.

Clotting profiles are obtained as a coagulation disorder is seen as an absolute contraindication unless corrected. An MRI is done prior to the procedure to help determine the more acute level causing the symptoms, as this may be problematic in patients with more than one affected vertebra on plain films. It is also important to exclude: (i) epidural or foraminal involvement; (ii) cord compression; (iii) tumour extension into the spinal canal or extensively into the pedicles; and (iv) other causes of pain.

Informed consent is obviously mandatory and we also like to have a family member present at this stage. The procedure, possible complications and expected outcome are discussed in detail. We tend to stress possible complications and downplay outcome as this helps in patient selection. Patients not experiencing severe pain often back down at this stage whereas patients with severe pain are less concerned.

\section{Contraindications}

Coagulation disorders are an absolute contraindication and need to be corrected prior to the procedure if possible. Neurological symptoms related to the compression by the abnormal vertebral body or tumour extension are also considered a contraindication to the procedure.

Extension and involvement of the posterior aspect of the vertebral body is seen as a relative contraindication. The more experienced interventional radiologists are doing these cases more frequently.

A neurosurgeon must be available in case emergency decompressive surgery is required.

\section{Technique}

The procedure is done on an outpatient basis. Prior to the procedure a single IV dose of antibiotics is given (e.g. $1 \mathrm{~g}$ of cefazolin/Ancef). The mixture of tobramycin with the cement has been advocated but is seldom used.

Conscious sedation is administered to the patient but more importantly good local anaesthetic needs to be given, with special attention being given to the periosteum. This is of more value than the IV sedation and we use Macaine because of its longer action. The patient is carefully monitored throughout the procedure. We have only done two patients under general anaesthesia, both of whom were experiencing severe pain from their metastatic lesions and could not lie still.

With the patient in the prone position access to the vertebral body is obtained via the pedicle under screening and CT guidance is not needed. ${ }^{1,8,9}$ Most centres use a bipedicular approach although one can also do a unipedicular puncture at a more acute angle, with the result that the tip of the needle is situated close to the midline of the vertebra. The needle is placed at the junction of the anterior one-quarter and posterior three- 


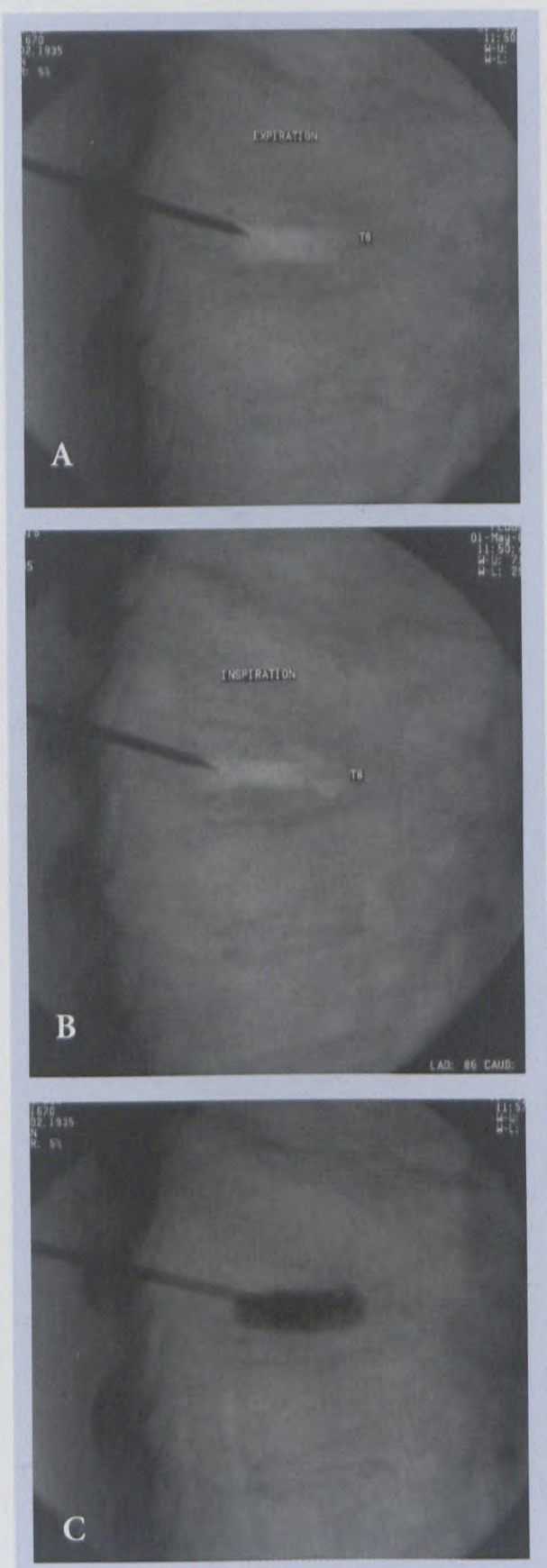

Fig. 1a,b,c. A patient with severe pain due to an osteoporotic fracture showing movement on breathing. Good filling is obtained with the patient being pain free after management.

quarters of the body (Fig. 1a,b,c). As the main venous channels of the vertebral body are located in the equatorial plain these should be avoided by inferior placement.

We use a bevelled $11 \mathrm{G}$ or $13 \mathrm{G}$ needle depending on the size of the verte- bra. This has two distinct advantages. Firstly one can guide the needle to a certain degree as the sharp end of the needle cuts into the bone and tends to guide the needle in that direction. For this reason we rotate the bevel to the outside when going through the pedicle so as not to penetrate the medial wall of the pedicle. Once one is through the pedicle and into the body it is advisable to rotate the bevel 180 degrees so that the sharp aspect of the bevel will guide the needle more to the centre of the body. The second reason for using a bevelled needle is that one can rotate it during injection of PMMA and this helps in guiding the flow of the cement.

Some centres do vertebral phlebography on all patients prior to administration of the PMMA. The rationale is that if the tip of the needle is in a big draining vein, one will need to advance the needle further forward to try and reposition outside the draining vein. Withdrawing the needle is not of help, as the contrast tends to follow the tract to the vein. If we are positioned in a large vein we do tend to advance the needle, but this is seldom of any help. What is important is to try to get a thicker consistency of PMMA to inject as this will not drain away as easily and basically embolises the proximal draining vein. The group at Johns Hopkins have done 205 consecutive cases without phlebography, and have not reported any serious complications. ${ }^{10}$ It is important to flush the contrast out of the needle with saline as it may otherwise be difficult to distinguish between contrast and PMMA at initial injection. The contrast sometimes also tends to pool in the vertebral body, making evaluation of cement injection more difficult. This contrast can also be washed out with some saline but does take a little longer and as one has limited time before the PMMA hardens, it is important not to mix the PMMA before one is ready to inject.

If no vertebral phlebogram was done one should still fill the needle with saline so as to prevent the possible complication of air embolism when injecting the cement.

Under optimal fluoroscopy the PMMA is injected into the body. The correct composition of the mixture with regard to the concentration of barium sulphate and PMMA is important, as this will affect the time the mixture takes to set. Previously these had to be mixed before administration, but currently there are products on the market which are ready and easy to use and are no more expensive than the original mixtures. With the current products one has about 10 minutes working time, which is ample. Cooling the liquid prior to mixing lengthens the time before setting and this may be useful when more than one level will be done. The consistency is roughly that of melted ice cream prior to the injection.

The cement is injected under lateral screening until it can be seen in the posterior part of the body (Fig. $2 \mathrm{a}, \mathrm{b}, \mathrm{c})$. The quality of the fluoroscopy unit is extremely important as visualisation of the cement during injection is one of the most important aspects of the procedure. Most of the serious complications documented have been associated with poor visualisation.

The patients are followed up for $3-4$ hours in the unit before being discharged home. They are phoned on day 1 and day 8 after the procedure. 

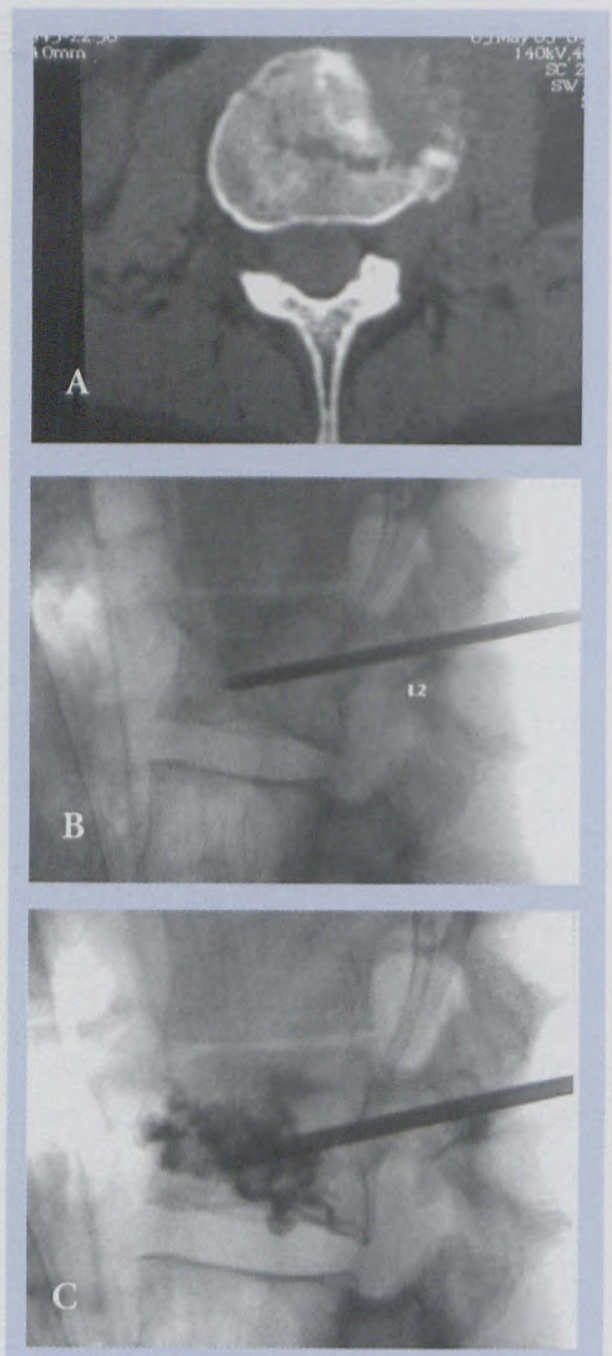

Fig. 2 a,b,c. A 39-year-old patient with colon carcinoma and a single metastatic deposit in the L2 vertebra. She was not a neurosurgical candidate and had already had radiotherapy. The patient had extreme pain and a unilateral approach was used for needle placement. Good vertebral filling was attained and the patient was pain free on day 2.

\section{Complications}

The most important part in preventing complications is in realising that pain relief is not related to the amount of cement injected. ${ }^{11}$ The complication rate is, however, directly related to the amount of PMMA injected. The rate of complications is very low and numerous series have not shown any complications.

Most serious complications are due to the leakage of contrast into the adjacent structures. Because this may lead to compression of nerve roots, etc. it must not be performed in a setting where no neurosurgeon is available for emergency decompressive surgery.

Clinically significant complications with vertebroplasty occur predominantly in patients with spinal metastases, but in most of these cases they resolve with medical treatment. ${ }^{8,912}$

In osteoporotic patients rib fractures have occasionally occurred due to the pressure needed to advance the needle through the pedicle.

Two deaths have been reported in the USA. In both instances the procedure was done under a $\mathrm{C}$-arm in theatre with the patient under general anaesthesia and seven or more levels were done. It is generally accepted that only under exceptional circumstances should more than two levels be done at a time.

One case of significant pulmonary embolism was reported in a 41-yearold female patient. She responded well on anticoagulant therapy. ${ }^{13}$

\section{Conclusion}

Vertebroplasty is a safe and successful method for the treatment of vertebral lesions due to osteoporotic fractures, metastatic lesions or haemangiomas.

Numerous studies have been done on the procedure and although relatively new in South Africa, it is well documented in Europe and the USA.
Strict adherence to the selection criteria and a multidisciplinary approach are needed.

The few complications reported have been associated with excessive PMMA injection, once again stressing the need for excellent imaging conditions.

\section{References}

1. Galibert P, Deramond H. Preliminary note on the treatment of vertebral angioma by percutaneous acrylic Vertebroplasty. Neurochirurgie 1987; 33: 166-168.

2. Barr JD, Barr MS, Lemley TJ, McCann RM. Percutaneous Vertebroplasty for pain relief and spinal stabilization. Spine 2000; 25: 923-928.

3. Deramond $\mathrm{H}$, Wright NT, Belkoff SM. Temperature elevation caused by bone cement polymerisation during Vertebroplasty. Bone 1999; 25: 17-21.

4. Cooper C, Athkinson E, Jacobsen S, Population-based study of survival after osteoporotic fractures. Am J Epidemiol 1993; 37.

5. Riggs BL, Melton LJ. The worldwide problem of osteoporosis: insights afforded by epidemiology. Bone 1995; 17: suppl, 505S-511S.

6. Martin JB, Sugio K, San Millian R, Murphy KJ, Piotin M, Rufenacht DA. Vertebroplasty: clinical experience and follow-up results. Bone 1999; 25: suppl, 11S-15S.

7. Murphy K, Deramond H. Percutaneous vertebroplasty in benign and malignant disease. Neuroimaging Clin N Am 2000; 3: 10.

8. Deramond H, Depriester C, Galibert P, Le Gars D. Percutaneous Vertebroplasty with polymethylmethacrylate. Technique, indications, and results. Radiol Clinic North Am 1998; 36: 533-546.

9. Cotton A, Boutry N, Cortet B, et al. Percutaneous Vertebroplasty: state of the art. Radiographics 1998; 18: 311-320: discussion 320-323.

10. Vasconceles C, Gailloud P, Beauchop N, et al. Is percutaneous Vertebroplasty without pre-treatment venography safe? Evaluation of 205 consecutive procedures. Am J Neuroradiol 2002; 23: 913-917.

11. Cotton A, Dewatre F, Cortet B, et al. Percutaneous Vertebroplasty for osteolytic metastases and myeloma: effects of the percentage of lesion filling and leakage of methyl methacrylate at clinical follow-up. Radiology 1996; 200: 525-530.

12. Chiras J, Depriester C, Weill A, et al. Percutaneous vertebral surgery. Techniques and indications. JNeuroradiol 1997: 24: 45-49.

13. Padovani B, Kasriel O, Brunner $\mathrm{P}$, et al. Pulmonary embolism caused by acrylic cement: a rare complication of percutaneous Vertebroplasty. Am J Neuroradiol 1999; 20: 375 377. 